\title{
Intra-cranial malignant peripheral nerve sheath tumor of olfactory nerve: a case report and review of literature
}

\author{
Varun Aggarwal, Amit Narang, Chandni Maheshwari, Divya Kavita \\ Baba Farid University of Health Sciences. Faridkot. Punjab, INDIA
}

\begin{abstract}
Malignant Peripheral Nerve Sheath Tumors (MPNSTs) are one of the very rare high grade malignancies usually affecting extremities or trunk. Incidence is 1/Lac. Intracranial MPNSTs are even rarer, schwannomatous and commonly affecting cranial nerves VIII \&VII). Intra-cranial MPNSTs are usually sporadic, arising de novo. The second most common mode of origin is from malignant transformation from pre-existing schwannomas or neurofibroma. We present an extremely rare and probably the first case of intra-cranial malignant peripheral nerve sheath tumor of the olfactory nerve in a non neurofibrosis patient with no prior history of irradiation.
\end{abstract}

Key words: Intra-cranial malignant peripheral nerve sheath tumor, Olfactory Nerve Tumor, MPNST

\section{Introduction}

Malignant peripheral nerve sheath tumor MPNSTs usually arise from peripheral nerves or shows differentiation towards nerve sheath cells. [9] MPNST of cranial nerves is very rare. The incidence of MPNST in the general population is $0.001 \%$ [8]. They constitute $5-10$ $\%$ of all soft tissue sarcomas and $50-70 \%$ are associated with Neurofibromatosis type 1 (NF1).[3,10] MPNSTs involving the Head and neck region comprise only $04-08 \%$ of all MPNSTs.[1] MPNST arising from olfactory nerve without any features of neurofibromatosis type 1 or type 2 as has been noted in our case. In the background of extensive literature search and with the best of our knowledge, probably this is the first case report of MPNST arising from olfactory nerve.

\section{Case history}

A 45 years old male presented to us with history of headache and bilateral loss of smell since six months and altered sensorium since seven days. On examination he had frontal lobe signs and features of foster Kennedy syndrome.

His Pre-operative MRI, T1 and T2 weighted images had $8 \times 7 \times 5 \mathrm{~cm}$ heterogeneous lesion originating from the anterior cranial fossa. On CEMRI (Figure 1) lesion was heterogeneously enhancing. His metastatic work up was negative. Patient was planned for 
surgery with diagnosis of atypical olfactory groove meningioma.

He underwent bifrontal craniotomy and gross total excision of the tumor (Figure 2).

Histopathology was suggestive of malignant peripheral nerve sheath tumor of the olfactory nerve. Post operatively patient was discharged on $7^{\text {th }}$ post op day with no fresh deficit. Patient underwent post op radiotherapy and is recurrence free since last six months.

\section{Discussion}

MPNSTs of the cranial nerves are rare tumors, and the literature is limited to isolated case reports and small case series. [9] In general population, the incidence of MPNST is 1/Lac population per year. [1] MPNSTs generally occur typically between ages 20-50 years whereas $10-20 \%$ of cases have been reported in the first 2 decades.[4,8] The common sites of origin include the extremities and trunk, usually sciatic nerve, brachial plexus and the sacral plexus MPNSTs of head and neck region comprise $04-08 \%$ of all MPNSTs and especially the cranial nerves involvement is extremely rare with only few cases or short case series found in the literature.[9] Most common intracranial MPNSTs are found in VIII \& V cranial nerves.[7] In retrospective analysis of intra cranial MPNSTs by Le'Hereux and Saliba which included 31 case reports and short case series comprising 60 cases, the incidence of cranial nerve MNSTs were: VIII CN-60\%; V CN-27\%, VII CN-10\%; III CN-6.7 \%; IV CN$1.7 \%$ \&VI CN-1.7 \%.[7] Etiologically, MPNST may occur infrequently when they arise de- novo, occur in association with neurofibromatosis (NF) [5,7], may be consequent to radiation (conventional or SRS) or arise as malignant transformation of schwannoma or neurofibroma.[7] MPNSTs are highly malignant tumors that grow rapidly along nerves, infiltrate surrounding tissues and develop hematogenous metastasis. MPNSTs may develop hematogenous distant metastases (most commonly to Lungs; other sites being Bones and Liver.[5]

MRI study does not allow confident distinction between benign and malignant nerve sheath tumors. A size more than $5 \mathrm{~cm}$, infiltrative margins and marked signal heterogeneity, adjacent structure compression and local invasion are features which favor MPNSTs. The diagnosis of MPNSTs necessitates biopsy and remains controversial due to its lack of specific morphological criteria and immunohistochemical or molecular tests. [6]

Treatment of MPST is primarily surgical. Gross total excision of intracranial MPNSTs significantly influences overall survival rates and relates inversely with local recurrence. Complete surgical tumor resection with preservation of neurological function should be the goal of the treatment. However, critical location and stubborn nature of tumor, complete surgical resection can be challenging and is not always feasible.[1,7] The role of adjuvant Radiotherapy remains controversial.[7] Numerous chemotherapy regimens have been used against MPNSTs but results have been unconvincing even when combined with surgery and radiotherapy. [3,7] In early stages of MNST Surgical resection of 
the tumor provides survival benefits. Whereas radiotherapy is useful in improving survival in metastatic MPNST. Combined therapy with surgery and radiotherapy are prognostically important in patient with tumors $>5 \mathrm{~cm}$. [2]

\section{Conclusion}

Intra-cranial olfactory MPNST is extremely rare. This is the probably first such reported case. Complete excision should be the goal of surgery which is the mainstay of treatment. However, due to the stubborn nature of tumor and intimate relation with critical neurovascular structures, this may not always be feasible. Combined therapy surgery with post op radiotherapy has some survival benefits in metastatic MPNST.

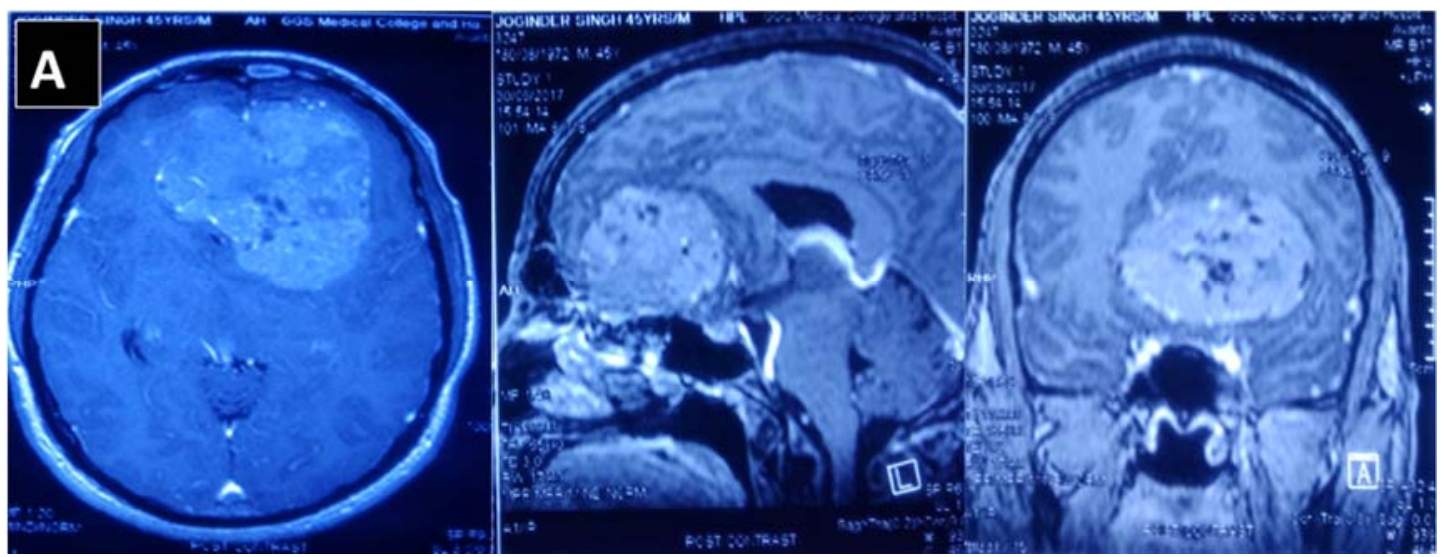

Figure 1 - Pre Op contrast enhance MRI brain (axial, sagittal and coronal view) Showing anterior cranial fossa SOL

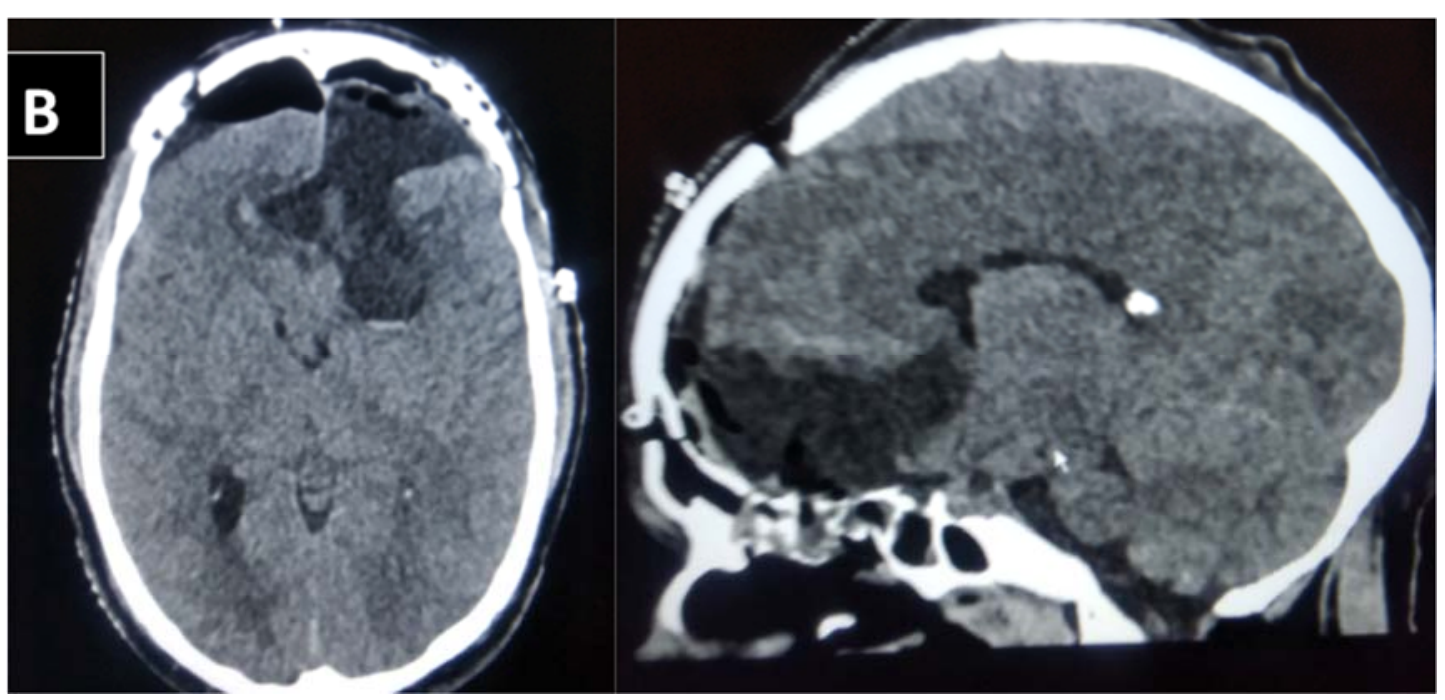

Figure 2 - Post op NCCT Head axial and (sagittal view) showing complete excision of the tumor 


\section{Correspondence}

Dr. Amit Narang

Email:drnarangamit@gmail.com

Assistant Professor, Neurosurgery, Guru Gobind

Singh Medical College and Hospital Faridkot,

Punjab. INDIA. 151203.

Phone 703836244

\section{References}

1.Anghileri M, Miceli R, Fiore M, Mariani L, Ferrari A, Mussi C, Lozza L, Collini P, Olmi P, Casali PG, Pilotti S, Gronchi A. Malignant peripheral nerve sheath tumors: prognostic factors and survival in a series of patients treated at a single institution. Cancer. 2006 Sep 1;107(5):1065-74.

2.Arshi A, Tajudeen BA, St John M. Malignant peripheral nerve sheath tumors of the head and neck: Demographics, clinicopathologic features, management, and treatment outcomes. Oral Oncol. 2015 Dec;51(12):1088-94.

3.Aydin MD, Yildirim U, Gundogdu C, Dursun O, Uysal $\mathrm{HH}$, Ozdikici M. Malignant peripheral nerve sheath tumor of the orbit: case report and literature review. Skull Base. 2004 May;14(2):109-13; discussion 113-4.

4.Ducatman BS, Scheithauer BW, Piepgras DG, Reiman HM. Malignant peripheral nerve sheath tumors in childhood. J Neurooncol. 1984;2(3):241-8.
5.Ferner RE, Gutmann DH. International consensus statement on malignant peripheral nerve sheath tumors in neurofibromatosis . Cancer Res. 2002 Mar 1;62(5):1573-7.

6.Kar M, Deo SV, Shukla NK, Malik A, DattaGupta S, Mohanti BK, Thulkar S. Malignant peripheral nerve sheath tumors (MPNST)--clinicopathological study and treatment outcome of twenty-four cases. World J Surg Oncol. 2006 Aug 22;4:55.

7.L'heureux-Lebeau B, Saliba I. Updates on the diagnosis and treatment of intracranial nerve malignant peripheral nerve sheath tumors. Onco Targets Ther. 2013 Apr 26;6:459-70

8.Panigrahi S, Mishra SS, Mishra S, Das S. Malignant peripheral nerve sheath tumor presenting as orbito temporal lump: Case report and review of literature. Asian J Neurosurg. 2016 Apr-Jun;11(2):170-1. doi: 10.4103/1793-5482.145065.

9.Scheithauer BW, Erdogan S, Rodriguez FJ, Burger PC, Woodruff JM, Kros JM, Gokden M, Spinner RJ. Malignant peripheral nerve sheath tumors of cranial nerves and intracranial contents: a clinicopathologic study of 17 cases. Am J Surg Pathol. 2009 Mar;33(3):32538

10.Wanebo JE, Malik JM, VandenBerg SR, Wanebo HJ, Driesen N, Persing JA. Malignant peripheral nerve sheath tumors. A clinicopathologic study of 28 cases. Cancer. 1993 Feb 15;71(4):1247-53. 